\title{
Role of bacteria in the pathogenesis and progression of acute and chronic lung infection
}

\author{
R A Stockley
}

The lung presents a major interface between the external environment and the body. Although antigens, including viable bacteria, are inhaled on a regular basis, health is maintained and the organisms are excluded by a multitude of sophisticated and complex host defence mechanisms. These include the integrity of the bronchial epithelium and the efficiency of the mucociliary escalator which prevents bacterial adherence and penetration whilst at the same time facilitating their removal. In addition, the lung secretions contain various proteins including lysozyme and lactoferrin which prevent bacterial replication, as well as immunoglobulin A which prevents bacterial adhesion and scavenging phagocytic cells to destroy the bacteria that remain.

The relationship between the lung and bacteria can take on several abstract forms: (a) a transient relationship where the host remains intact, the bacterial load remains low, and the primary lung defences outlined above result in effective clearance; (b) acute infections where the bacterial load is higher, the local defences fail to cope, and bacterial numbers increase. At this point an acute inflammatory process occurs leading to the recruitment of the secondary lung defence systems including scavenging neutrophils. The patient becomes acutely unwell and the inflammatory response results in sterilisation of the bronchial tree and the infective process resolves; (c) colonisation: this usually occurs on the background of abnormal host defence. The process is low grade with a mild degree of inflammation and a balance is established between the local host defence mechanisms and bacterial replication to achieve a position of stability. This situation may be associated with recurrent acute exacerbations which are self-limiting and resolve; and (d) chronic infection: in this situation the bacterial load is sufficient to generate a marked inflammatory response that fails to clear the organism. It can become self-perpetuating and lead to the development of chronic progressive bronchial disease.

The evidence for, and understanding of, these mechanisms is based on very carefully controlled animal experiments of acute and chronic infection together with transient observations in human subjects. ${ }^{2}$ The exact mechanisms involved remain to be clarified and much of our understanding of pathogenesis is based upon in vitro studies utilising pure cytokines and studying their effects. In vivo the situation may be much more complex, particularly since a "cocktail" of cytokines is released at the same time, some of which have diametrically opposed effects in vitro. Nevertheless, with this as a background it is possible to derive some concepts of the processes involved and some of the mediators that may be relevant.

\section{Studies in man}

The importance of the primary and secondary host defence in the relationship between the lung and bacteria can only be determined by indirect observations. Genetic disorders of mucociliary clearance (the major primary host defence) are known to be associated with the development and progression of chronic lung infection. In addition, systemic immune deficiency and disorders of neutrophil function (secondary host defence factors) are also associated with persistent recurrent infections. These defects clearly emphasise the importance of host defence. However, most patients with both acute and chronic infections do not appear to have any major defect of host defence that has yet been identified. ${ }^{34}$

\section{ACUTE INFECTION/PNEUMONIA}

This is a systemic illness in which the primary lung defence has been overcome by the inhaled bacteria. Secondary host defence mechanisms are activated with neutrophil infiltration and inflammation develops within the lung. These episodes are usually short lived and may be either fatal, self-limiting, or curtailed by the use of the appropriate antibiotic therapy.

The process of acute infection should theoretically go through several stages. Once the inflammatory process has started there is likely to be an amplification stage which will result in either sufficient or insufficient recruitment of secondary host defences. If the response is sufficient the bacteria will be destroyed, the process will be switched off, and resolution will take place. On the other hand, if the amplification process is insufficient progressive illness may develop until antibiotic therapy 


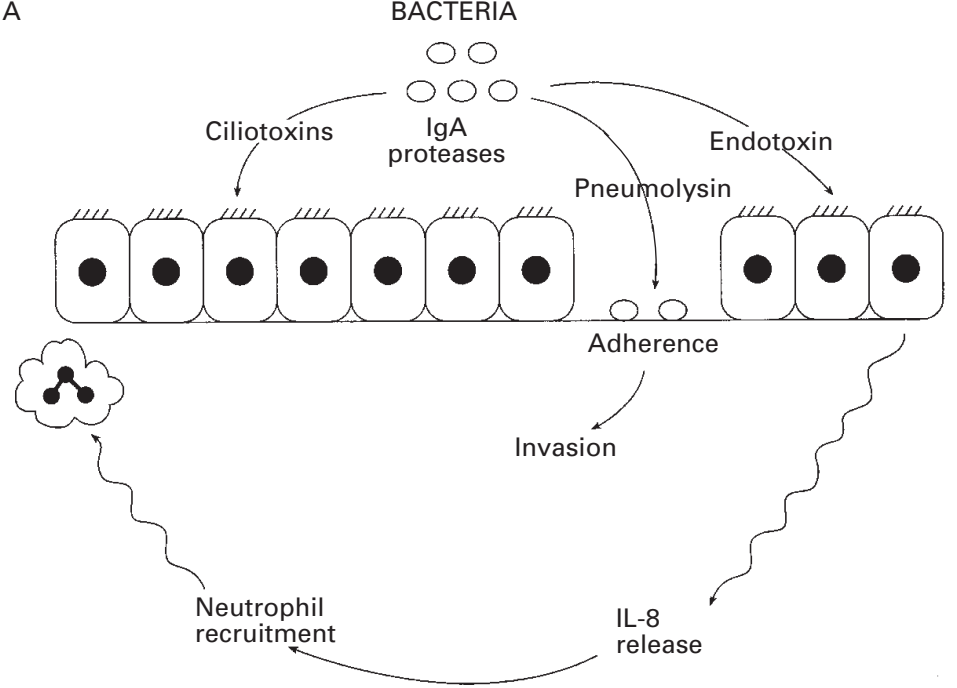

B

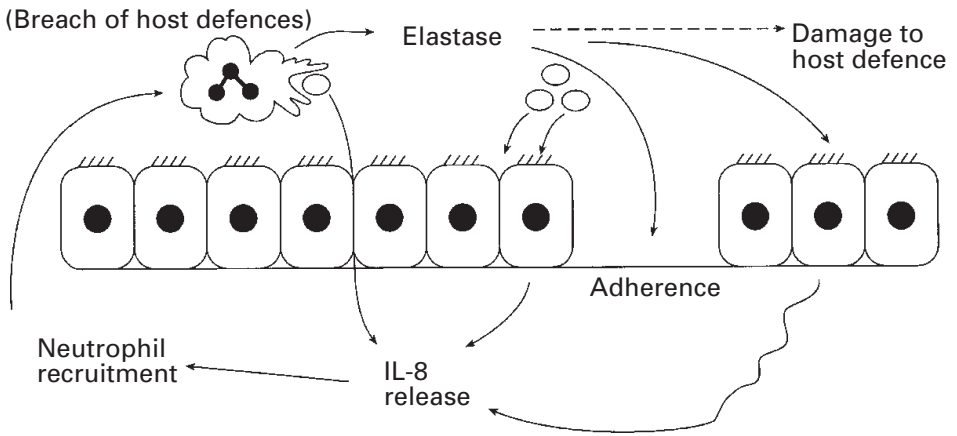

C

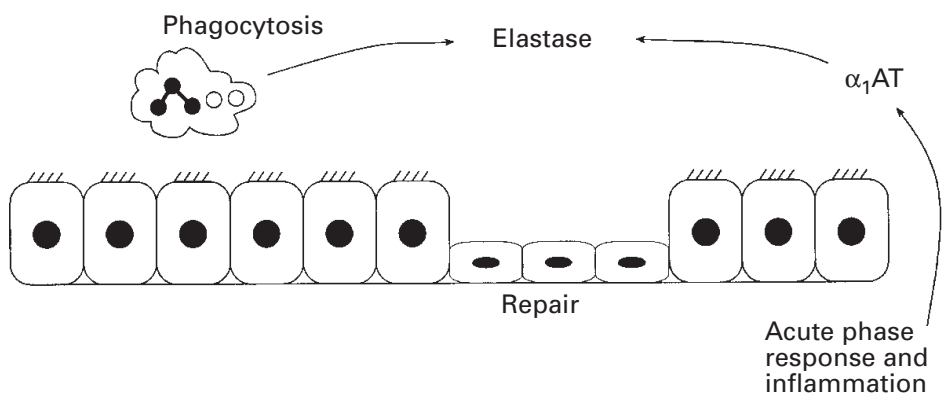

Figure 1. (A) Initiation of the inflammatory process. Inhalation of a significant number of bacteria will lead to damage within the airway by the release of factors such as cilia toxins, pneumolysin, and endotoxin. In addition the release of IgA proteases interferes with the function of local IgA. Stimulation of the epithelial cells by endotoxin results in a release of many pro-inflammatory cytokines including interleukin-8 which is responsible for neutrophil recruitment. (B) Amplification of this initial response. Activated neutrophils within the airway continue to phagocytose bacterial particles and the activation of the cells leads to further release of interleukin-8. In addition, elastase release from the neutrophil also has the potential to damage the epithelium as well as various other host defence factors (see text). The activity of the elastase can cause further interleukin-8 release, adding to the chemotactic gradient resulting in continuing neutrophil recruitment. (C) Resolution. Complete phagocytosis of all the bacteria has taken place, reducing the effect of bacterial factors on host defences and interleukin-8 production by epithelial cells. In addition the inflammatory response results in an increase in proteinase inhibitors such as $\alpha_{1}$-antitrypsin in the airway which can inactivate the elastase and thereby all of its effects on host defences and epithelial tissues. As the drive to neutrophil recruitment and the loss of epithelial damaging factors decreases, epithelial repair can take place.

in conjunction with host defences results in bacterial destruction and subsequent resolution. These simple steps of initiation, amplification and resolution are outlined in fig 1.
Initiation

The initiation of the infective process occurs when sufficient bacteria are present in the lung and overcome the ability of local host defences to clear them. This may simply involve the inhalation of sufficient bacteria in the first instance, or may be potentiated by the release of various bacterial factors that interfere with host defences such as pyocyanin and hydroxyphenazine which significantly reduce mucociliary clearance, ${ }^{5}$ lipopolysaccharide which damages epithelium, ${ }^{6}$ or IgA proteases ${ }^{7}$ which damage secretory IgA and therefore may facilitate bacterial adherence to the epithelium.

At this point the inflammatory process is initiated. Evidence has shown that bacteria possess the ability to stimulate human bronchial epithelial cells to produce a variety of cytokines some of which may be pro-inflammatory. Of major importance is thought to be the chemoattractant interleukin 8 (IL-8) and tumour necrosis factor $\alpha(\mathrm{TNF}-\alpha){ }^{8}$ Epithelial IL-8 is a major neutrophil chemoattractant and, once released from the bronchial epithelium, it forms a diffusion gradient into the circulation attracting and activating nearby leucocytes. The process of neutrophil migration will be further enhanced by stimulation of endothelial cell adhesion molecules and, although some of these are constitutionally expressed on the endothelium, the release of pro-inflammatory cytokines such as TNF- $\alpha$ will increase the expression of these molecules ${ }^{9}$ and facilitate neutrophil adhesion.

\section{Amplification}

The recruitment of neutrophils to the airways may actually facilitate the amplification process. Activation of neutrophils has been shown to release both $\mathrm{LTB}_{4}{ }^{10}$ and IL- $8^{11}$ which will add to the chemoattractant concentration in the airway and potentiate the diffusion gradient into the circulation. In addition, release of neutrophil elastase as the neutrophil becomes activated has also been shown to increase epithelial cell IL-8 production, ${ }^{12}$ again adding to the chemoattractant gradient.

\section{Resolution}

This whole process may be limited by a successful and adequate host response. Providing there is a sufficient degree of bacterial phagocytosis the process will become self-limiting. The mechanisms involved in switching off the host defence have been least studied. Simplistically the resolution process could take place if the bacterial load is reduced and this switches off the bacterial stimulus to the inflammatory phase (the contribution to IL- 8 and TNF production). This in itself will reduce the drive to neutrophil recruitment. As fewer neutrophils are recruited and activated they will generate less IL-8 and $\mathrm{LTB}_{4}$ in their own right, as well as less neutrophil elastase to promote IL-8 production by the epithelial cell. This change in itself may be sufficient to switch off the inflammatory process but may be facilitated by the generation of anti-inflammatory cytokines. 
At this point the acute phase response may also be important. Alpha-1-antitrypsin undergoes a 3-4-fold rise in serum concentrations during episodes of systemic inflammation. This, coupled with the increase in lung endothelial and epithelial permeability that occurs during infection, should result in a major increase in the diffusion of this proteinase inhibitor into the lung. The effect would be to limit the amount of active neutrophil elastase within the lung tissue and it may therefore serve as a mechanism for downregulating the inflammatory response being driven by the release of this enzyme. In addition, one of the other acute phase proteins, $\alpha_{1}$-antichymotrypsin, has been shown to be a major inhibitor of neutrophil chemotaxis. ${ }^{13}$ Again, the concentration of this protein rises dramatically during inflammation (several fold within 12 hours). The effect of this also could limit neutrophil traffic into the lung and thereby downgrade the inflammatory process.

Finally, during the process of resolution, apoptosis of the recruited neutrophils may be an important mechanism. How this takes place and its relationship to the initiation of the inflammatory process is difficult to discern. TNF- $\alpha$ has been shown to increase apoptosis in vitro ${ }^{14}$ and may therefore be important in the resolution of inflammation. However, as indicated above, the same cytokines may actually accentuate the inflammatory process by the stimulation of endothelial cell adhesion molecules. Furthermore, activation of neutrophils by endotoxin can prevent the effect of TNF on apoptosis. ${ }^{14}$ Thus, changes in the biochemical environment in the airway may affect the way the neutrophil responds to single or multiple factors. Similarly, studies have shown that IL-6 is also capable of promoting apoptosis $^{15}$ and yet studies by Khair and colleagues have indicated that IL-6 is released at the same time as pro-inflammatory cytokines from bronchial epithelium exposed to endotoxin from Haemophilus influenzae. ${ }^{8}$ The rationalisation of these diverse properties and their relationship to the initiation and resolution of inflammation remains to be achieved.

\section{COLONISATION}

Colonisation represents a state between acute infection and complete resolution. It is generally associated with an identified breach in host defences and usually this is because of a defect in mucociliary clearance related to ciliary dysfunction, epithelial destruction, or abnormal mucus production. A failure of this important mechanism of clearance would, firstly, delay removal of bacteria from the lung and, secondly, provide a suitable culture medium for bacterial replication. Chronic colonisation therefore reflects a state in which bacterial replication keeps pace with the primary host defence clearance mechanisms.

There may be a low grade inflammatory process in patients with chronic bronchitis and emphysema in the stable clinical state. The patients may often have a significant bacterial load in their secretions $\left(10^{8}\right.$ viable organisms/ $\mathrm{ml}$ ) plus a small number of recruited neutrophils and detectable plasma protein transudation into the secretions. Nevertheless, this represents virtually a symbiotic relationship. The bacteria isolated are usually those considered to be of low pathogenicity (Haemophilus influenzae, Moraxella catarrhalis, Haemophilus parainfluenzae) and may themselves facilitate this whole process by interfering with several mechanisms of host defence. For instance, bacterial products have been shown to interfere with neutrophil chemotactic responses ${ }^{16}$ and may therefore downregulate an important mechanism that would lead to sterilisation of the bronchial tree. In addition, bacteria have been shown to have a major effect on factors involved in mucociliary clearance. Cilia toxins have been shown to be produced by a variety of bacteria which colonise the airways in patients with chronic bronchial disease. In addition, bacteria can lead to excess mucus production in their own right ${ }^{17}$ and will therefore lead to chronic bronchitis. Bacterial products can cause epithelial damage ${ }^{6}$ which will facilitate bacterial adherence and again lead to a reduction in their clearance. Finally, pathogenic bacteria are known to produce IgA proteases ${ }^{7}$ which may therefore interfere with the ability of IgA to prevent bacterial adherence, again a process that would facilitate bacterial persistence in the airways.

It is possible that the process itself may be perpetuated by host immune defects. As indicated at the beginning of this article, major defects of immunity are associated with persistent and/or recurrent bacterial infections. However, most patients with chronic bronchial disease do not have a clearly identifiable immune defect. Nevertheless, there are studies which suggest that in some patients the immune response may be abnormal, resulting in the production of the wrong type of antibody that does not facilitate bacterial phagocytosis and clearance but rather acts as a blocking antibody, "protecting" the bacteria from normal clearance mechanisms. ${ }^{18}$

In addition, recent studies have suggested that the bacteria may be able to facilitate their survival by continually changing their antigenic nature to remain ahead of a host immune response. ${ }^{19}$ The importance of this mechanism in the persistence of bacteria in the airways of patients with bronchial disease and the initiation of acute exacerbations of chronic bronchitis is clearly worthy of further study.

\section{CHRONIC INFECTION}

Chronic bronchial infection appears to be associated with an overactive response of the immune system (which may indeed be inappropriate) and the perpetuation of the inflammatory response. Studies have shown that such patients often have increased circulating immunoglobulin levels and increased immunoglobulins in the secretions. ${ }^{4}$ The lung tissues contain increased numbers of immunoglobulin generating $\mathrm{B}$ cells, ${ }^{20}$ particularly the $\operatorname{IgA}_{1}$ and IgA $_{2}$ subclasses. ${ }^{21}$ In addition, the $\mathrm{T}$ cell population is increased with the greatest increase 


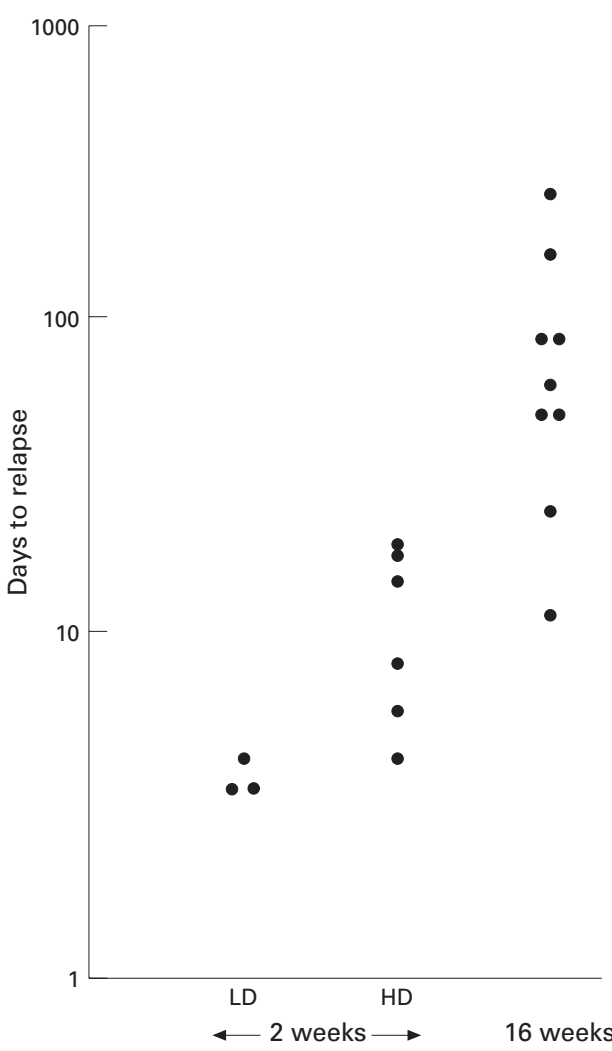

Figure 2 Effect of antibiotics on relapse from chronic infection in bronchiectasis showing the number of days from cessation of effective antibiotic therapy to the chronic infective state. Each point indicates the results of an individual patient. The results are for patients who have been treated with either two weeks of low dose (LD) antibiotic therapy (250 $\mathrm{mg}$ amoxycillin three times a day) or high dose (HD) therapy ( $3 \mathrm{~g}$ amoxycillin twice daily), or four months continuous therapy with amoxycillin given in effective doses orally or by the inhaled route. Published with permission from Stockley. ${ }^{22}$

being in the $\mathrm{T}_{4}$ suppressor subset. ${ }^{20}$ The lung tissues and airways contain large numbers of neutrophils as part of the inflammatory response, but in this situation neutrophil recruitment and activation is persistent and does not get switched off unless successful antibiotic intervention occurs, and even then the response may be short lived. ${ }^{22}$

The processes involved can only be alluded to. The inhalation of large numbers of bacteria would initiate the inflammatory process. The amplification stage should take place normally but the response itself may be insufficient to clear bacteria which continues the amplification process (see fig 1). Alternatively, the response may be excessive which in itself may be counterproductive, destructive, and facilitate bacterial survival, thereby perpetuating the process. In this respect the role of the neutrophil has been implicated as a major factor. ${ }^{1}$ Neutrophil recruitment and activation results in the release of a variety of tissue damaging substances including neutrophil proteinases and oxidants. These may interfere with host defences in various ways including reduction of mucociliary clearance, excess mucus production, mucus gland hyperplasia, bronchial epithelial damage (facilitating bacterial ad- herence), damage to immunoglobulins (preventing opsonisation), and even destruction of phagocytic receptors on the neutrophil itself (reducing opsonophagocytosis). This sequence of events has led to the concept of neutrophil mediated progression of chronic bronchial disease and persistence of chronic infection. ${ }^{1}$ As intimated above, the process can often be reversed by the use of appropriate antibiotics. However, such responses are usually short lived and patients may require long term antibiotic therapy in order to downregulate the inflammatory process and prevent persistent bronchial disease. In some instances this process may be permanently reversed, such that withdrawal of antibiotic therapy after a period of time may not be associated with an immediate return to the chronic infective state (fig 2).

In conclusion, bacteria play a role in initiating the processes involved in both acute and chronic lung infection. The former are usually short lived episodes which generally result in resolution and the return to a state of health. In the latter case the infective process, although relatively confined to the lung, may persist and be associated with processes that perpetuate and in most severe cases be related to progression of the chronic lung disease.

1 Stockley RA. Bronchiectasis: new therapeutic approache based on pathogenesis. Clin Chest Med 1987;8:481-94.

2 Stockley RA, Hill SL, Morrison HM. Effective antibiotic treatment on sputum elastase in bronchiectatic outpatients in a stable clinical state. Thorax 1984;39:414-9.

3 Stanley PJ, Korbo G, Cole PJ. Serum IgG subclasses in chronic and recurrent respiratory infection. Clin Exp Immunol 1984;58:703-8.

4 Bilton D, Leung AYT, Hill SL, Stockley RA. Defective lung defences. Eur Respir 7 1992;9:159-65.

5 Wilson R, Pitt T, Taylor G, et al. Pyocyanin and 1-hydroxy phenazine produced by Pseudomonas aeruginosa inhibit the beating of human respiratory cilia in vitro. $\mathcal{F}$ Clin Invest 1987;79:221-9.

6 Wilson R, Sykes D, Rutman A, et al. The effect of Haemophilus influenzae lipopolysaccharide on human respiratory epithelium in vitro. Thorax 1986;41:728-9.

7 Kilian M, Mestecky J, Kullhevy R, et al. IgA proteases from Haemophilus influenzae, Streptococcus pneumoniae, Neisseria meningitidis and Streptococcus sanguis: comparative immunochemical studies. F Immunol 1980;124:2596-600.

8 Khair OA, Devalia JL, Abdelaziz MM, et al. Effect of Haemophilus influenzae endotoxin on the synthesis of IL6 , IL-8, TNF- $\alpha$ and expression of ICAM1 in cultured human bronchial epithelial cells. Eur Respir f 1994;7: 2109-16

9 Wardlaw A. Leukocyte adhesion to endothelium. Clin Exp Allergy 1990;20:619-26.

10 Ford-Hutchinson AW, Ray MA, Doig MV, et al. Leukotriene B4 - a potent chemokinetic and aggregating substance released from polymorphonuclear leukocytes. Nature 1980;286:264-5.

11 Takehashi GW, Andrews DF, Lilly MB, et al. Effect of granulocyte-macrophage colony-stimulating factor and interleukin-3 on interleukin-8 production by human neutrophils and monocytes. Blood 1993;81:35764.

12 Nakamura H,Yoshimura K, McElvaney NG, Crystal RG. Neutrophil elastase in respiratory epithelial lining fluid of individuals with cystic fibrosis induces interleukin-8 gene expression in the human bronchial epithelial cell line. $f$ Clin Invest 1992;89:1478-84

13 Lomas DA, Stone SR, Llewellyn-Jones CG, et al. Control of neutrophil chemotaxis by inhibitors of cathepsin $\mathrm{G}$ and chymotrypsin. F Biol Chem 1995;270:23437-43.

14 Hachiya O, Takeda Y, Miyata H, Watanabe H, Yamashita T, Sendo F. Inhibition by bacterial lipopolysaccharide of spontaneous and TNF-alpha-induced-human neutrophil apoptosis in vitro. Microbiol Immunol 1995;39:715-23.

15 Afford SC, Pongracz J, Stockley RA, et al. The induction by human Interleukin- 6 of apoptosis in the promonocytic by human Interleukin-6 of apoptosis in the promonocytic 267:21612-6.

16 Cundell DR, Taylor GW, Kanthakumar K, et al. Inhibition of human neutrophil migration in vitro by low-molecularmass products of non typeable Haemophilus influenzae. Infect Immun 1993;61:2419-24.

17 Li JD, Dohrman AF, Gallup M, et al. Transcriptional activation of mucin by Pseudomonas aeruginosa lipopolysaccharide in the pathogenesis of cystic fibrosis lung disease. Proc Natl Acad Sci 1997;94:967-72. 
18 Fick RB, Olchowski J, Squier SU, et al. Immunoglobulin G subclasses in cystic fibrosis, $\mathrm{IgG}_{2}$ response to Pseudomonas lipopolysaccharide. Am Rev Respir Dis 1986;133:418-22. 19 Groenveld J, van Alphen L, Eijk PP, et al. Changes in outer membrane proteins of non-typeable Haemophilus influenzae in patients with chronic obstructive pulmonary disease. F Infect Dis 1988;158:360-5.

20 Lappe E, Silva JR, Jones JAH, Cole PJ, Poulter LW. The immunological component of the cellular inflammatory infiltrate in bronchiectasis. Thorax 1989;44:668-73.

21 Burnett D, Crocker J, Stockley RA. Cells containing IgA subclasses in bronchi of subjects with and withou chronic obstructive lung disease. F Clin Pathol 1987;40 1217-20.

22 Stockley RA. Bronchiectasis - a management problem? $\mathrm{Br}$ 